\title{
NUMERICAL INVESTIGATION OF STRESSES IN STEEL PLATE USING LAMB WAVES
}

\author{
Atef Eraky \\ Professor, Structural Engineering, Civil Engineering Department, \\ College of Engineering, Zagazig University, Egypt
}

Walid El-Deeb

Assistant Professor, Electronics \& Communications Engineering Department, College of Engineering, Zagazig University, Egypt

\section{Shimaa Emad}

Assistant Lecture, Civil Engineering Department, College of Engineering, Zagazig University, Egypt

\begin{abstract}
This paper detects, through numerical simulations, the stress in structures using lamb waves. The finite element model of steel plate was developed using the commercial finite element programs ABAQUS and ANSYS. Dynamic models were run for Lamb wave propagation in steel plate under the effect of different compressive and tensile stresses. The simulations have revealed that with the variation of stress there is no effect of time of flight. In the case of compressive stress, it's found that the amplitude of received wave is increased slightly with increasing of compressive stress. In otherwise of tensile stress, the amplitude of received wave is also decreased slightly with increasing of tensile stress.
\end{abstract}

Keywords: Stress, Acoustoelasticity and Lamb wave

Cite this Article: Atef Eraky, Walid El-Deeb and Shimaa Emad, Numerical Investigation of Stresses in Steel Plate using Lamb Waves International Journal of Civil Engineering and Technology, 11(8), 2020, pp. 24-36.

https://iaeme.com/Home/issue/IJCIET?Volume $=11 \&$ Issue $=8$

\section{INTRODUCTION}

Detection of stress in structural elements especially in vital structures such as bridges is very important because stress concentration can cause damage or failure of the structure. Ultrasonic stress measurement is one of the non-destructive evaluation (NDE) methods that depends on acousto-elastic theory [1]. Measurement of stresses using non-destructive technology provides early indications of failure without destroying the structures [2]. Ultrasonic and X-rays are the main methods of non-destructive evaluation that can reveal 
substantial subsurface flaws in materials. Other techniques such as magnetic particle eddy current, and neutron diffraction can also detect some subsurface features but only near the surface of the materials. However these techniques are hazardous, time-consuming and limited to laboratory applications [3-4]. Ultrasonic waves are the sound waves propagating in a medium with frequency greater than $20 \mathrm{kHz}$. The ultrasonic waves are classified as either guided waves such as Lamb waves, or bulk waves, which are pressure and shear waves [5]. Non-destructive evaluation (NDE) and structural health monitoring (SHM) interested in guided waves in plates that are known as Lamb waves due to their ability to propagate relatively for long distances with maintaining to their sensitivity to damage [6-8]. Gandhi, N and et al de-veloped the theory of acoustoelastic Lamb wave propagation for isotropic media subjected to a biaxial, homogeneous stress field [6]. They found that, as expected, dispersion curves change anisotropically for most stresses, modes, and frequencies. Interestingly, for some mode-frequency combinations, changes in phase velocity are isotropic even for a biaxial stress field. Mosbuth, $\mathrm{M}$ and et al also studied the effect uniaxial stress on the propagation of higher-order Lamb wave modes [9]. The outcomes of this study can be utilized in the development of new techniques for the measurement of the applied stresses based on the acoustoelastic effect. Qui, L and et al proposed the multiphysics simulation method of Lamb wave propagation with piezoelectric transducers under load condition [7]. Comsol multiphysics is used to simulate the propagation of Lamb wave in the aluminum plate.

In this research, ABAQUS and ANSYS finite element programs are used to simulate steel plates under compressive and tensile stresses to study the wave propagation in stressed plates.

\section{ACOUSTOELASTIC THEORY}

In general, the acoustic wave time of flight (TOF) through a fixed distance is calculated by dividing the wave propagation distance by the wave velocity. But when the material is applied to compressive strength or tensile stress, the change in wave propagation velocity is slightly, also the time of wave propagation will change, this is called acoustic- elastic law. As shown in Equation (1) from the changes in the transit time, the load stress can be estimated as follows [3]:

$$
\frac{\Delta V}{V_{0}}=1+C_{A} V_{0} \sigma
$$

where, $\triangle \mathrm{V}$ is the change in the wave velocity due to stress, $\mathrm{V}_{0}$ is the wave velocity in unstressed specimen and $\mathrm{C}_{\mathrm{A}}$ is the acousto-elastic coefficient and $\sigma$ is the stress in the specimen. Abbasi, $\mathrm{Z}$ and Ozevin, D [4] used the acoustoelasticity theory to investigate the influence of ultrasonic frequency on shear stress measurement. This study investigated the effect of sensor frequency on stress measurement. Rayleigh wave penetration depth which is approximately one wavelength decreases as the selected frequency increases.

\section{LAMB WAVE PROPAGATION}

Guided wave propagation in plates has a type of waves called Lamb waves that characterized by its long-range propagation and high sen-sitivity to damage, so it's used in non-destructive evaluation (NDE) and structural health monitoring (SHM). Lamb waves have two modes, symmetric (S) and anti-symmetric. These modes depended on wave propagation in the thickness direction. Lamb waves have a dispersal property as the variation of their speed depending on their frequencies and plate thickness. Figure (1) show the propagation of Lamb waves in the pre-stressed plate with thickness d. Direction $\mathrm{x} 1$ is the direction of initial prestress and also the direction of Lamb wave propagation. Direction $\mathrm{x} 3$ is the direction of plate thickness [10]. 


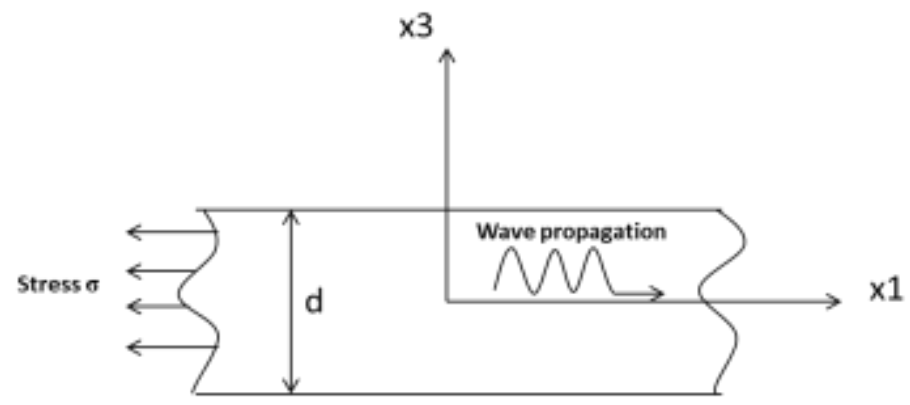

Figure 1 Lamb wave propagation [10].

From the previous study the equation of motion of the wave (incremental deformation $u$ ) under the assumption of homogenous material and the existence of a quasi-static predeformation expressed in terms of the natural (unstressed) coordinates ( $\mathrm{x}$ ) is provided as

$$
A_{i j k l} \frac{\partial^{2} u k}{\partial x_{j} \partial x_{i}}=\rho^{0} \frac{\partial^{2} u_{i}}{\partial t^{2}}
$$

where $\rho^{0}$ is the density in the natural state, and the coefficient Aijkl is given by the following equation

$$
A_{i j k l}=C_{i j k l}+C_{j l m n} e_{m n}^{i} d_{i k}+C_{i j m l} e_{k m}^{i}+C_{m j k l} e_{i m}^{i}+C_{i j k l m n} e_{m n}^{i}
$$

where $\mathrm{e}_{\mathrm{mn}}^{\mathrm{i}}$ is the Lagrangian infinitesimal strain tensor in the initial state, and $\mathrm{C}_{\mathrm{ijkl}}$ is the stiffness tensor constituting the stress-strain relationship in the initial state and it can be expressed as

$$
T_{i j}^{i}=C_{i j k l} e_{k l}^{i}
$$

where $\mathrm{T}_{\mathrm{ij}}^{\mathrm{i}}$ is the second Piola-Kirchhoff stress tensor in the initial state. Similarly, the incremental stress-strain relationship can be written as [6].

$T_{i j}=B_{i j k l} \frac{\partial u_{k}}{\partial x_{l}}$

where

$$
B_{i j k l}=C_{i j k l}+C_{i j m l} e_{k m}^{i}+C_{i j k l m n} e_{m n}^{i}
$$

Acousto-elastic Lamb-wave propagation in the pre-stressed plate can be analyzed by solving Eq. (1) subject to the stressfree boundary conditions at $x 3= \pm d / 2$ and the incremental stress given by Eq. (4). First, the form of the solution is assumed as

$$
u_{j}=U_{j} e^{i k\left(x_{1}+\alpha x_{3}-c t\right)}
$$


where $\mathrm{k}$ is the wave number in the $x_{1}$ direction, $\mathrm{c}$ is the phase velocity, and $\alpha$ is the wave number ratio of $x_{3}$ to $x_{1}$. Substitution of Equation (7) into Equation (2) yields the Christoffel equation.

$$
K_{m n} U_{n}=0
$$

For a nontrivial solution for $U_{n}$ to exist, $\operatorname{det}\left(K_{m n}\right)$ must be zero, and it produces the following equation as a function of $\alpha$

$$
P_{6} \alpha^{6}+P_{4} \alpha^{4}+P_{2} \alpha^{2}+P_{0}=0
$$

where $P_{6}, P_{4}, P_{2}$ and $P_{0}$ are the coefficients derived in [6]. The $\alpha\left(\alpha_{q}, q=1 \ldots 6\right)$ values physically represent three upward and three downward bulk wave. The displacement field induced by Lamb waves can be expressed as the sum of the displacements of these six bulk waves as

$$
\left\{u_{1}, u_{2}, u_{3}\right\}=\sum_{q=1}^{6}\left\{1, V_{q}, W_{q}\right\} U_{1 q} e^{i k\left(x_{1}+\alpha_{q} x_{3}-c t\right)}
$$

where $V_{q}$ and $W_{q}$ are the displacement ratios of $U_{2}$ and $U_{3}$ to $U_{1}$, respectively, and derived as

$V_{q}=\frac{U_{2 q}}{U_{1 q}}, W_{q}=\frac{U_{3 q}}{U_{1 q}}, q=1 \ldots 6$

The stress field is obtained by substituting Equation (11) into Equation (5) to obtain

$$
\left\{T_{33}, T_{13}, T_{23}\right\}=\sum_{q=1}^{6} i k\left\{D_{1 q}, D_{2 q}, D_{3 q}\right\} U_{1 q} e^{i k\left(x_{1}+\alpha_{q} x_{3}-c t\right)}
$$

where

$$
\begin{aligned}
& D_{1 q}=B_{3311}+B_{3312} V_{q}+\alpha_{q} B_{3333} W_{q}, \\
& D_{2 q}=\alpha_{q}\left(B_{1313}+B_{1323} V_{q}\right)+B_{1331} W_{q}, \\
& D_{3 q}=\alpha_{q}\left(B_{1323}+B_{2323} V_{q}\right)+B_{1332} W_{q},
\end{aligned}
$$

Applying the stress free boundary conditions at $\mathrm{x}_{3}={ }_{-} \mathrm{d} / 2$

$$
D_{11} G_{1} \cot \left(\gamma \alpha_{1}\right)+D_{13} \cot \left(\gamma \alpha_{3}\right)+D_{15} \cot \left(\gamma \alpha_{5}\right)=0
$$

And

$$
D_{11} G_{1} \tan \left(\gamma \alpha_{1}\right)+D_{13} \tan \left(\gamma \alpha_{3}\right)+D_{15} \tan \left(\gamma \alpha_{5}\right)=0
$$

where $\gamma=k d / 2=\omega d / 2 c$, in which $\omega$ is the angular frequency. The coefficient of $G_{m}$ can be written as 


$$
\begin{aligned}
& G_{1}=D_{23} D_{35}-D_{33} D_{25}, \\
& G_{3}=D_{31} D_{25}-D_{21} D_{35}, \\
& G_{5}=D_{21} D_{33}-D_{31} D_{23}
\end{aligned}
$$

\section{NUMERICAL SIMULATION}

In this paper, finite element programs are used to simulate steel plates under compressive and tensile stresses to study the wave propagation in the stressed plates. Two programs ABAQUS and ANSYS are used to study the effect of various stresses on the time of flight of the wave and on its amplitude.

\subsection{ABAQUS Modeling}

3D ABAQUS model is used, to model the wave propagation in stressed steel plate. The steel plate dimensions are $400 \mathrm{~mm}$ length, $200 \mathrm{~mm}$ width and $2 \mathrm{~mm}$ thickness. The plate is fixed at one end and loaded at the other end as shown in figure (2). The material properties of the steel plate are listed in the table (1). Two steps are performed to model the wave propagation in the stressed plate. The first step is stationary to model the compressive or tensile stress on the plate. The stress distribution in the plate in this step is shown in figure (3). The second step is ABAQUS explicit dynamic to simulate the Lamb wave propagation in the plate under compressive or tensile stress as shown in figure (4). The plate has been performed with the solid cubic particles and the homogenized model has meshed with an 8-nodes brick element. The element mesh size depends on the wavelength and the mesh size taken as $1 \mathrm{~mm}$ and the corresponding time step 1e-7 s.

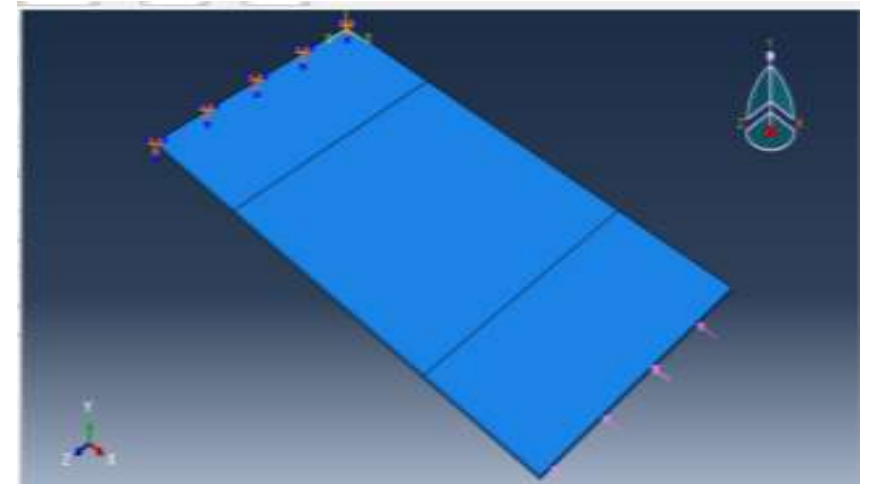

Figure 2 the steel plate modeling.

Table 1 material properties of the steel plate.

\begin{tabular}{|c|c|c|c|}
\hline Material & $\begin{array}{c}\text { Young } \\
\text { modules } \\
(\mathbf{N} / \mathbf{m} 2)\end{array}$ & Poisson's ratio & $\begin{array}{c}\text { Density } \\
(\mathbf{K g} / \mathbf{m} 3)\end{array}$ \\
\hline steel & $2 \mathrm{E} 11$ & 0.3 & 7850 \\
\hline
\end{tabular}




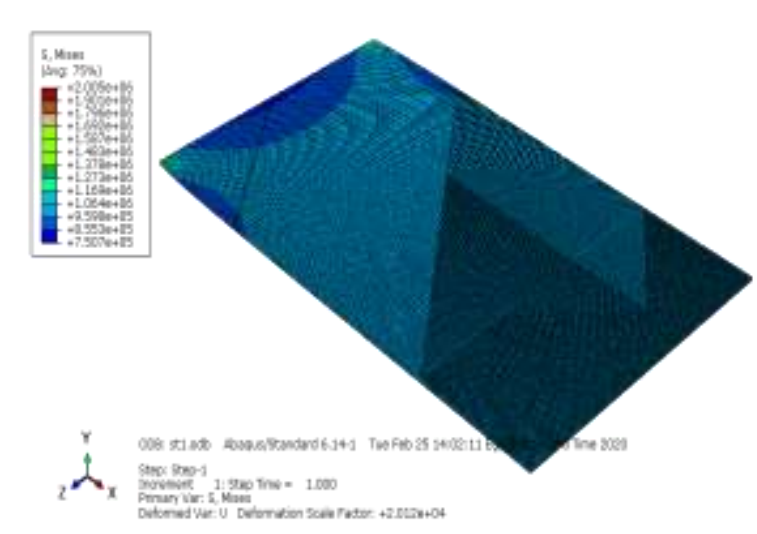

Figure 3 stress in steel plate in the stationary step.

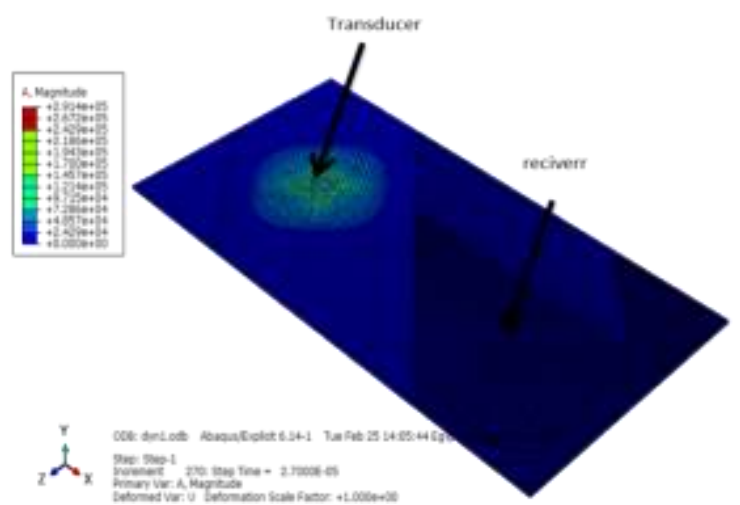

Figure 4 Wave propagation in the steel plate.

Hanning window with five cycles which is expresses in Equation (17) and shown in figure (5) is used as a force function to generate lamb wave in ABAQUS.

$$
E=A[1-\cos (2 \pi f t / N)] \sin (2 \pi f t)
$$

where $\mathrm{A}$ is taken $50, f$ is the frequency and takes as $200 \mathrm{KHz}, \mathrm{N}$ is the number of cycles $\mathrm{N}=5$ and $\mathrm{t}<\mathrm{N} / f$.

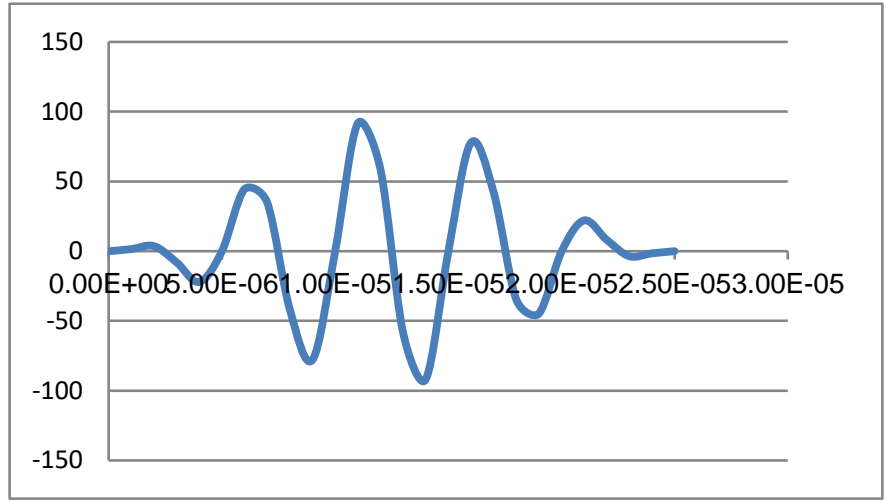

Figure 5 Hanning window excitation signal.

In this study, compressive or tensile stresses with different values as $1 \mathrm{MPa}, 20 \mathrm{Mpa}$, $40 \mathrm{MPa}, 60 \mathrm{MPa}, 80 \mathrm{MPa}$ and $100 \mathrm{MPa}$ are used to investigate lamb wave characteristic under stress. The lamb wave is modeled as point load with the amplitude of Hanning window function in the transducer point and the results are investigated at the receiver point as shown in figure (4).

\subsubsection{Compressive Stress}

Lamb wave propagation in steel plate under compressive stress is studied with different values of stresses as mention in the previous section. Figure (6) shows the acceleration response of the receiver point for different compressive stress values. There is no clear difference between the acceleration response for different compressive stresses. Figure (7) shows the zoomed amplitude of the acceleration at the first peak. 
Atef Eraky, Walid El-Deeb and Shimaa Emad

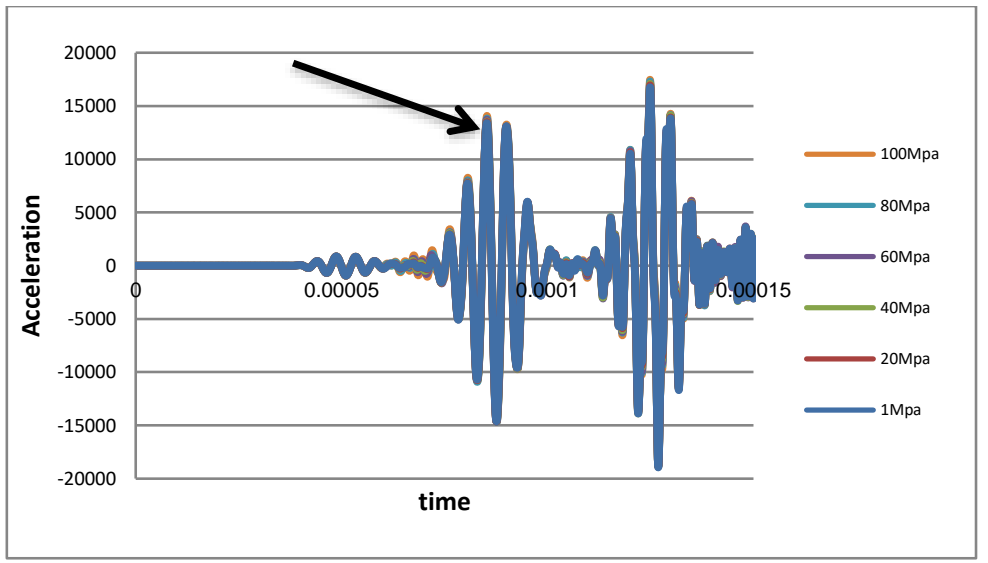

Figure 6 Acceleration response at the received point

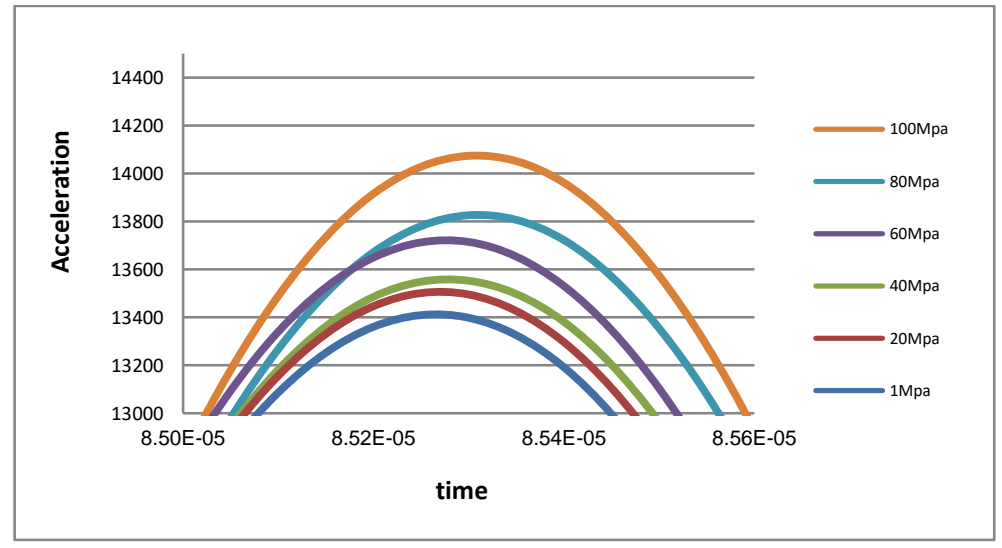

Figure 7 Zoomed First Peak

It is shown that there is no variation in the time of flight of the wave with the change in the stress and it is also shown that with increasing compressive stress, the acceleration amplitude increases due to the space between particles in the plate decreases under the compressive stress. The amplitude of the peaks for different compressive stress is fitted as shown in figure (8).

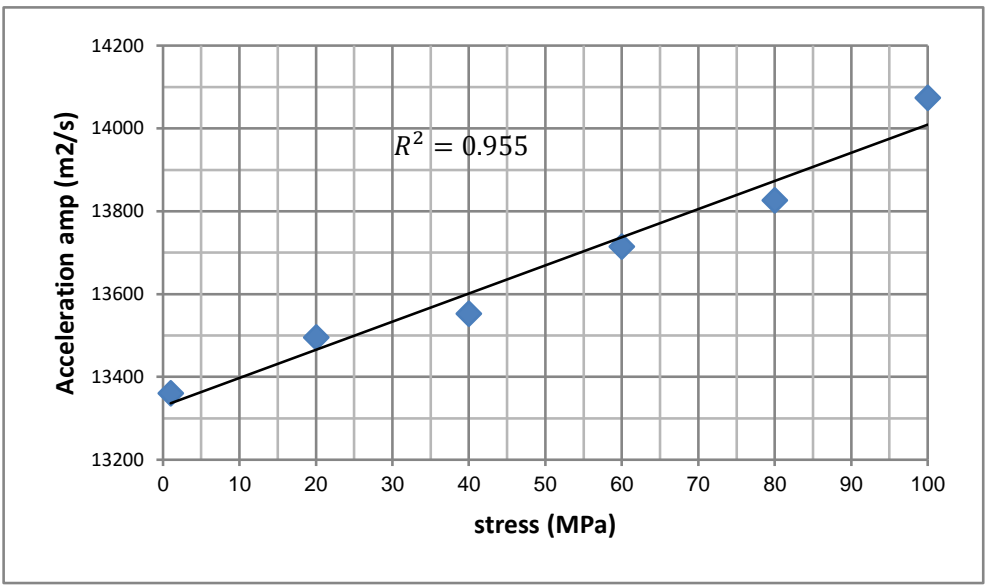

Figure 8 Variation of acceleration amplitude with compressive stress 


\subsubsection{Tensile Stress}

Lamb wave characteristic is investigated at the received point in the steel plate under different tensile stress ranges from $1 \mathrm{MPa}$ up to $100 \mathrm{MPa}$. Figure (9) shows the acceleration time history at the received point with different tensile stress and figure (10) shows the zoomed amplitude of the first peak. It is shown that the increase of tensile stress doesn't make any variation in time of flight of the lamb wave but the amplitude of the received wave decreasing with increasing tensile stress as shown in figure (10). The peak acceleration amplitude is fitted with the tensile stress as shown in figure (11). The amplitude of received wave decreases due to that increasing tensile stress increases the space between steel plate particles and then leads to decreasing the received wave amplitude.

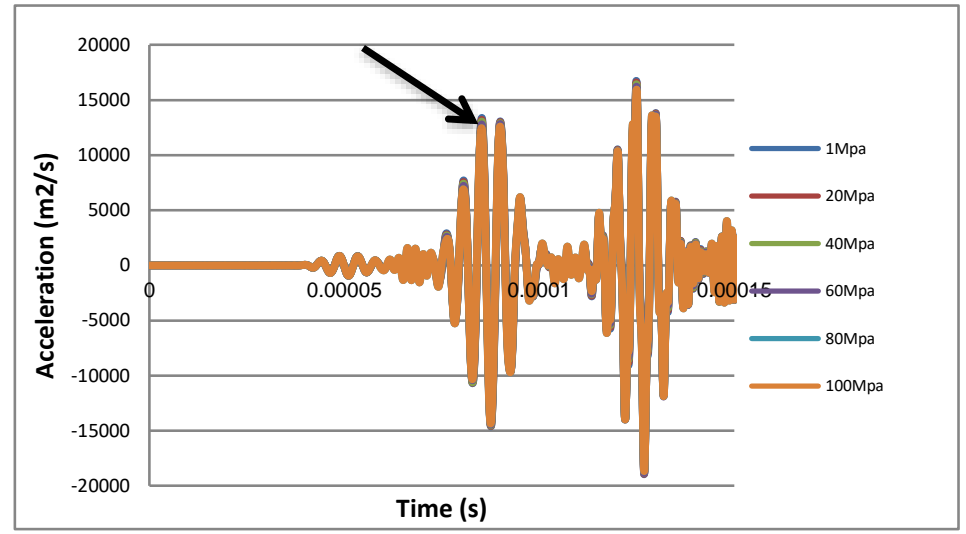

Figure 9 Acceleration response at the received point

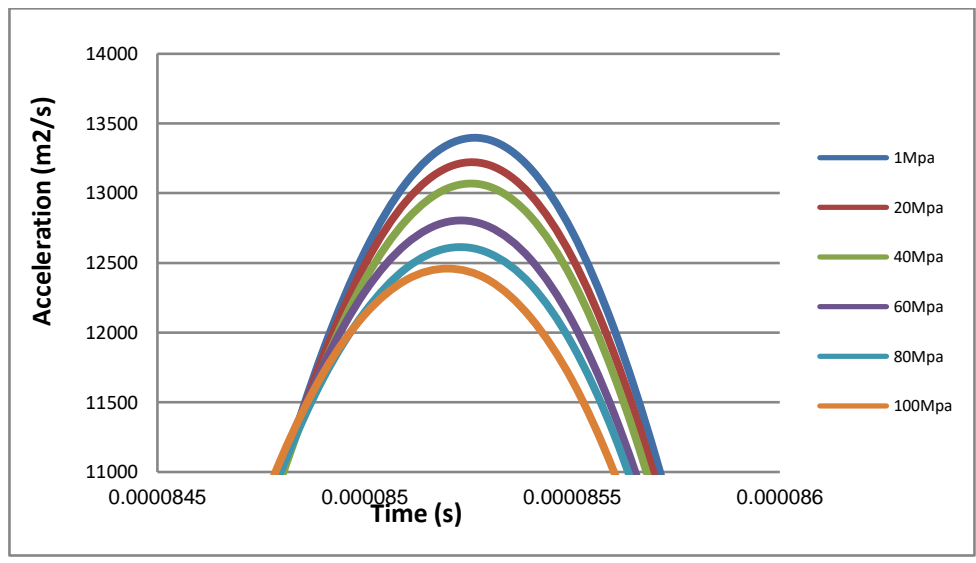

Figure 10 Zoomed at amplitude

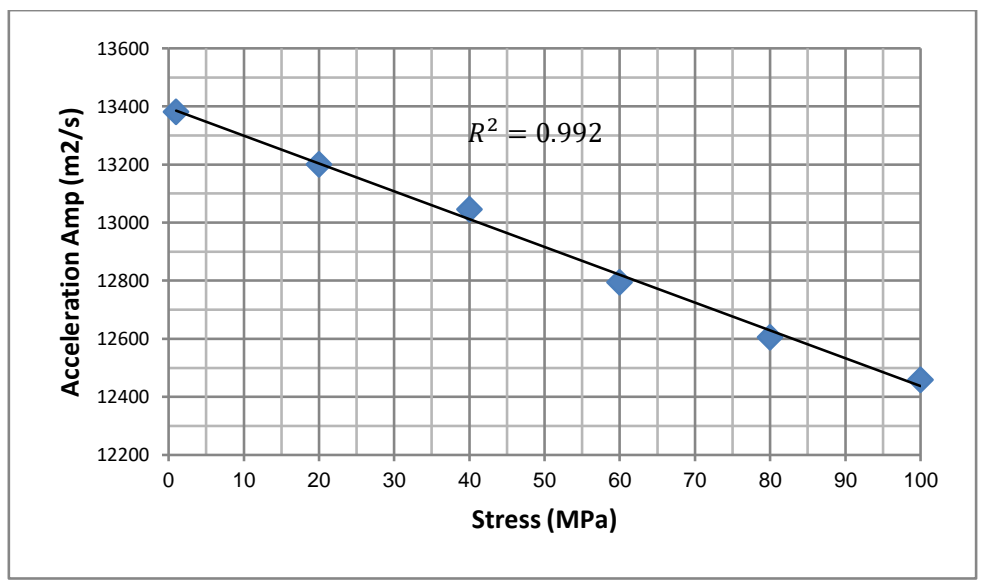

Figure 11 Variation of acceleration amplitude with compressive stress 
Figure (12) shows the relation between peak amplitude of the received wave and the compressive and tensile stress.

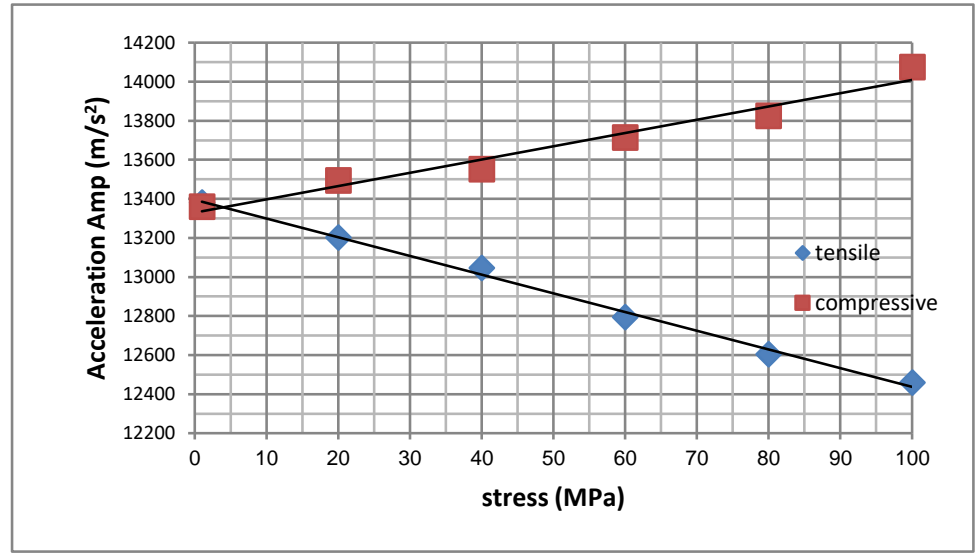

Figure 12 Variation of acceleration amplitude with compressive and tensile stresses

\subsection{ANSYS modeling}

ANSYS software is used to model lamb wave propagation in the stressed steel plate. The steel plate dimension and its properties are the same that used in ABAQUS software. Also the wave excitation signal, element size, and time step are the same. To model Lamb waves in stressed steel plate, two steps are used; static and dynamic explicit as shown in figure (13).

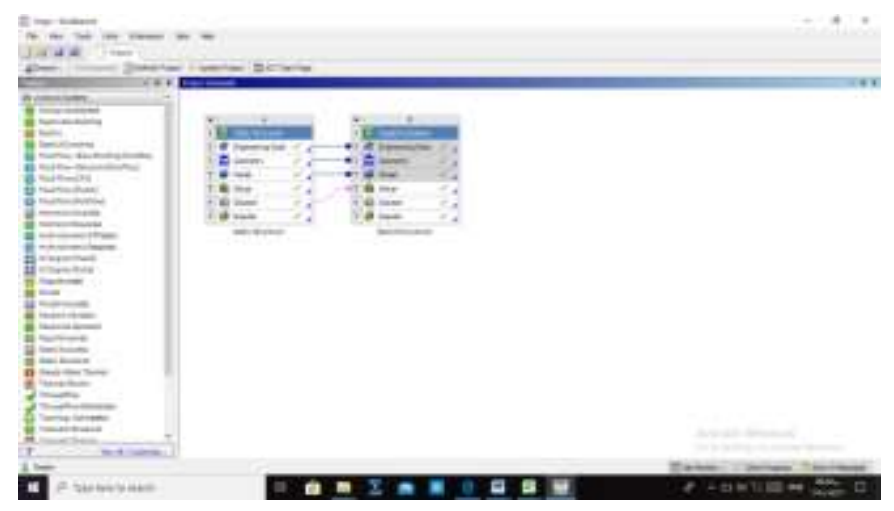

Figure 13 ANSYS program steps

Figure (14) shows the stress distribution in the steel plate in the first static step due to the pressure load at its free end with $1 \mathrm{MPa}$.

Figure (15) shows the wave propagation in the stressed steel plate at time 1e-4 second.

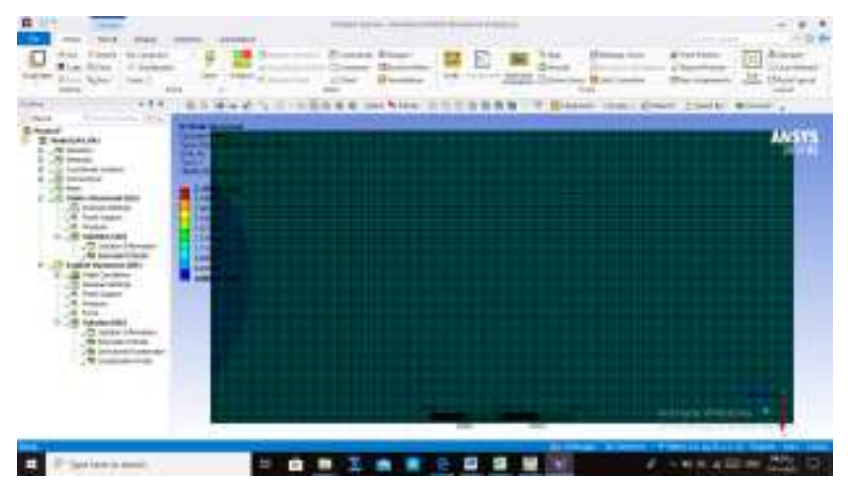

Figure 14 stress distribution in steel plate 


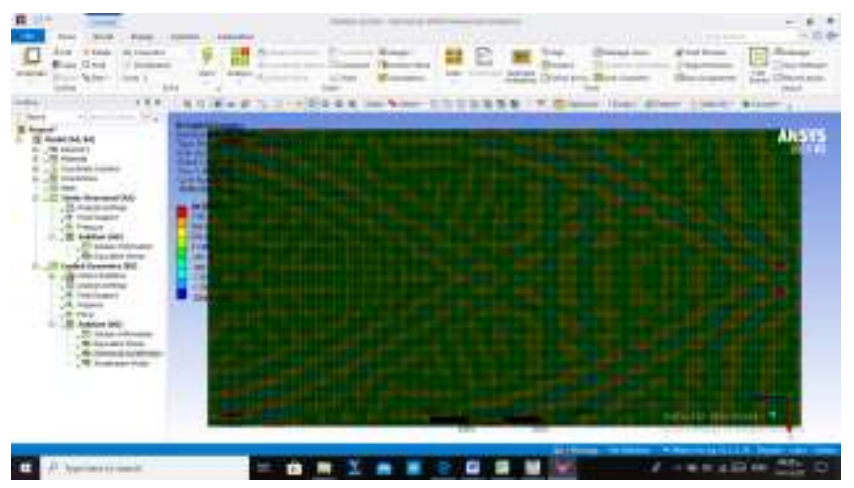

Figure 15 Wave propagation in steel plate at time 1E-4

\subsubsection{Compressive Stress}

Different compressive stresses are applied to the free end of the plate, and then the wave is excited at the transducer point and the acceleration is measured at the receiver point. The acceleration time history at the receiver point is shown in figure (16), and zoomed in figure (17).

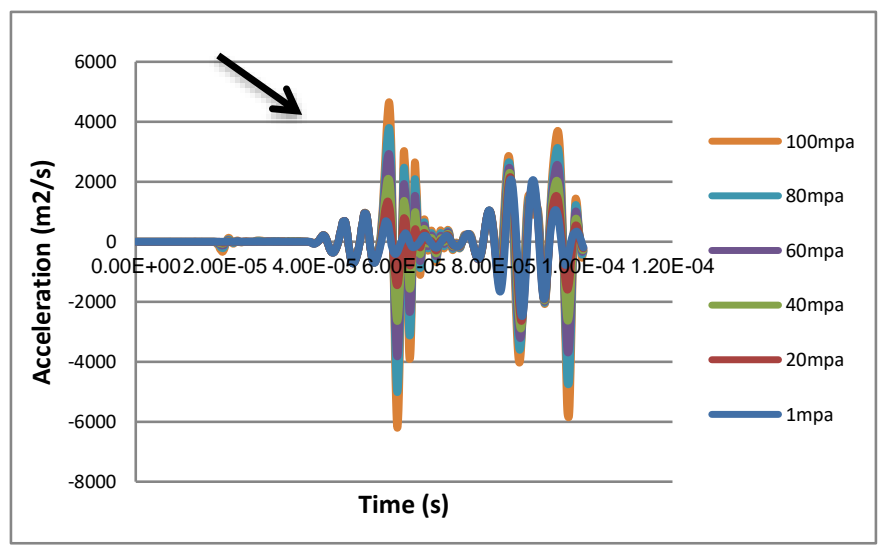

Figure 16 Acceleration at the received point for different compressive stresses

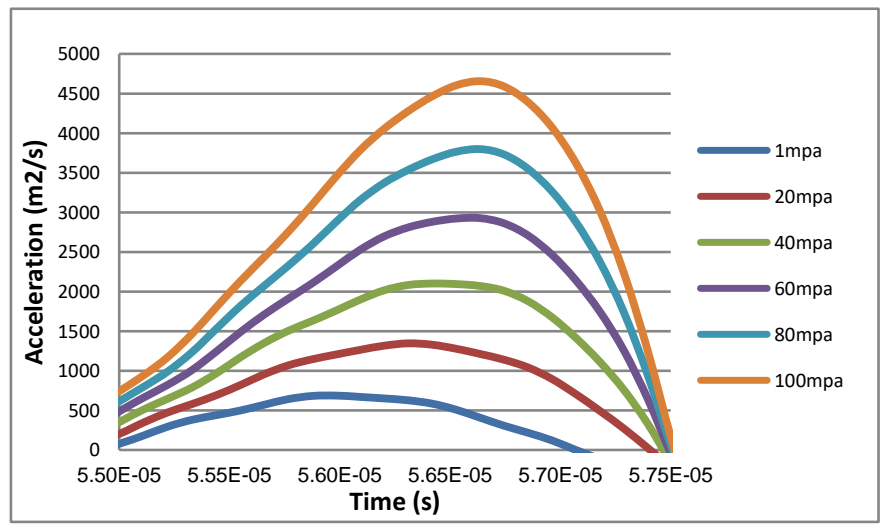

Figure 17 Zoomed acceleration peaks

It is shown that the time of flight doesn't change with the change of the applied stresses, while the acceleration amplitude increases with increasing compressive stress. This relation between the peak acceleration amplitude and the applied compressive stresses is shown in figure (18). 


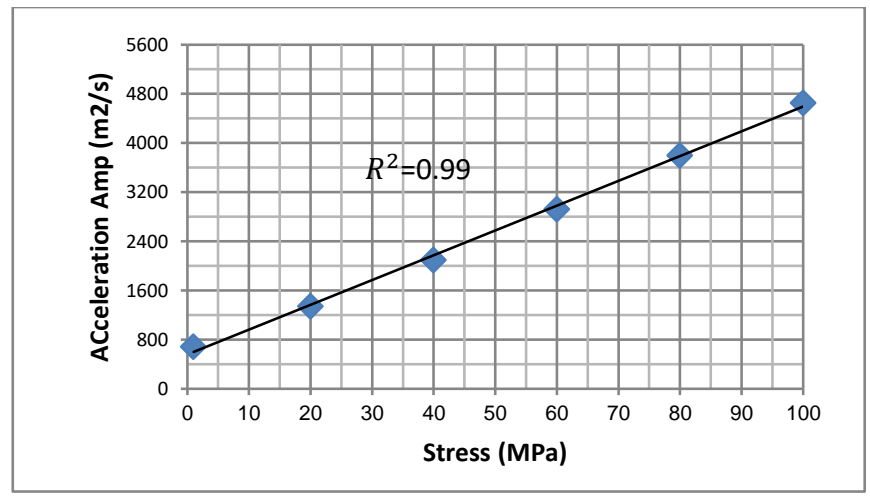

Figure 18 Variation of acceleration amplitude with compressive stress

\subsubsection{Tensile Stress}

In this part tensile pressure is applied at the free end of the plate in the static step and then wave signal is excited at the transducer point in the dynamic explicit step. The acceleration at the receiver point is monitoring at different values of pressure load as shown in figure (19), and zoomed as shown in figure (20).

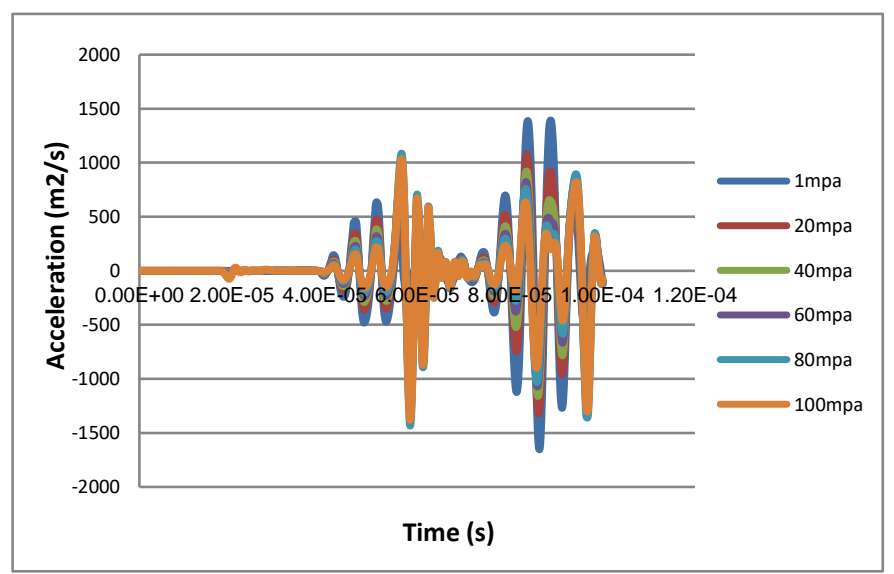

Figure 19 Acceleration at the received point for different tensile stresses

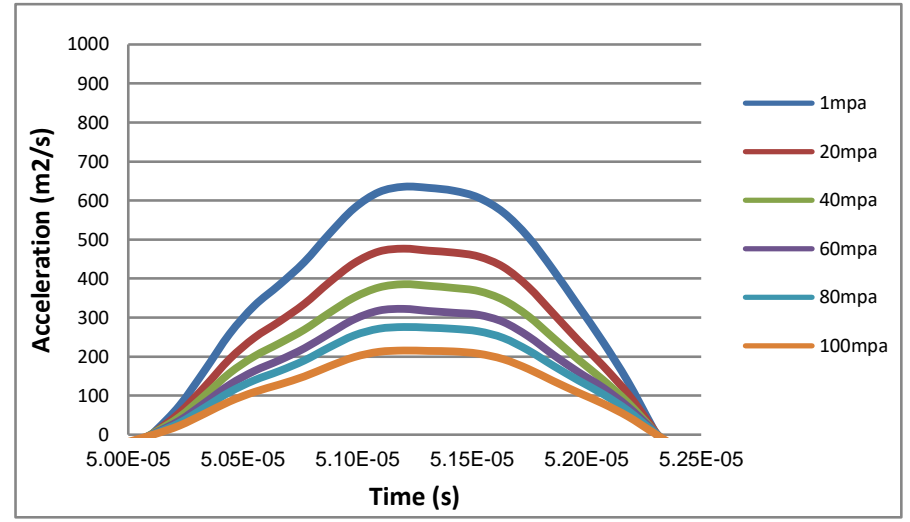

Figure 20 Zoomed first peak acceleration amplitude.

The peak acceleration amplitude is fitted with the tensile stress as shown in figure (21). The fitted peak acceleration amplitude for different compressive and tensile stresses are shown in figure (22). 
Numerical Investigation of Stresses in Steel Plate using Lamb Waves

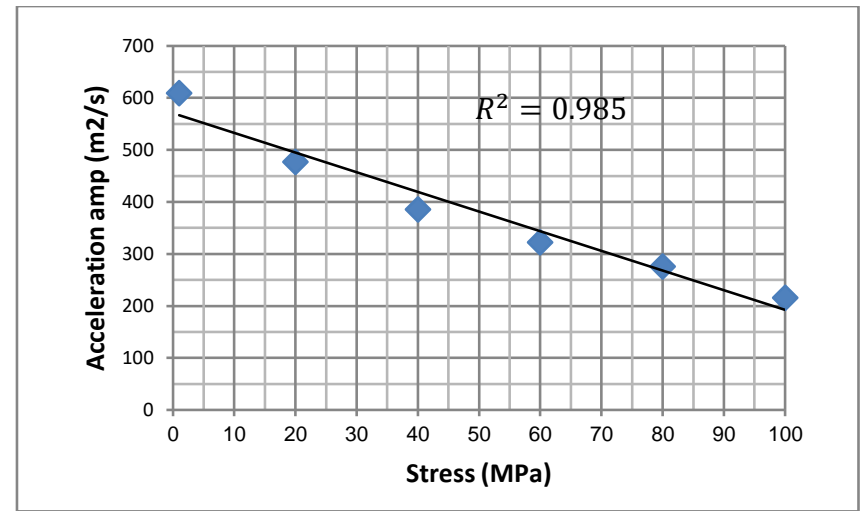

Figure 21 Variation of acceleration amplitude with tensile stress.

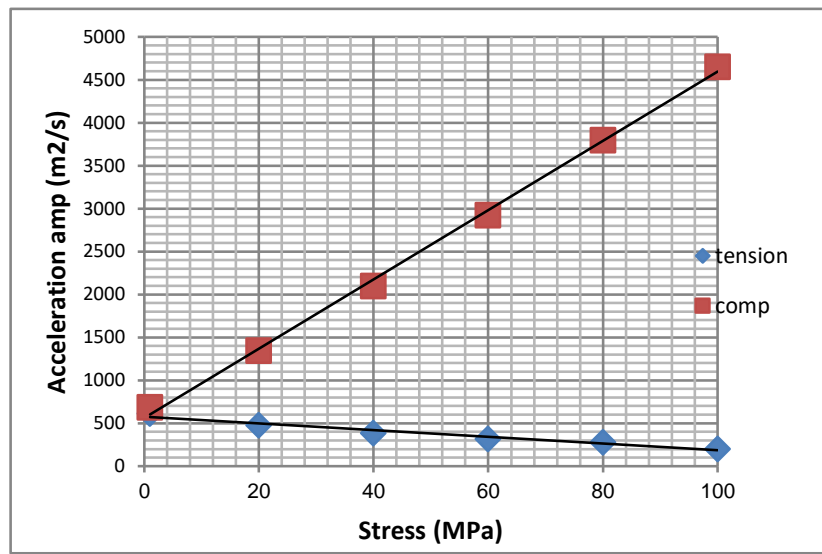

Figure 22 Variation of acceleration amplitude with compressive and tensile stresses.

\section{CONCLUSIONS}

In this research, ABAQUS and ANSYS finite element programs are used to simulate steel plates under compressive and tensile stresses to study the wave propagation in stressed plates. Many conclusions are withdrawn from the results as follows;

- There are no variations in time of flight of the wave with variation in stress value in steel plate.

- Acceleration amplitude due to lamb waves is increased with increasing compressive stress in steel plate.

- Acceleration amplitude due to lamb waves is decreased with increasing tensile stress in steel plate.

- The relation between the acceleration maximum amplitude and the applied tensile or compressive stress is approximately linear.

- ANSYS results are more clarify than ABAQUS results.

\section{REFERENCES}

[1] Achenbach, J.D and Rajapakse, Y.," Solid mechanics research for quantitative non-destructive evaluation" Martinus Nijhoff Publishers, Dordrecht 1987.

[2] Post,A,M., " Non -destructive measurement of stress using ultrasonic leaky lamb waves" A thesis submitted to the Faculty of Graduate Studies York university Toronto, Ontario (2008). 
[3] Hussin, M., Chan, T., Fawzia, S., and Ghasemi, N., "Finite Element Modelling of Lamb Wave Propagation in Prestress Concrete and Effect of the Prestress Force on the Wave's Characteristic" 10th RMS Annual Bridge Conference: Bridges - Safe and Effective Road Network, 2 - 3 December 2015, Ultimo, N.S.W. https://eprints.qut.edu.au/92622/.

[4] Abbasi, Z., Ozevin, D., "The influence of ultrasonic frequency on shear stress measurement using acoustoelasticity" AIP Conference Proceedings, 1706,070010 (2016).http://doi.org/10.1063/1.4940528.

[5] Hussin, M. K., "Identification of prestress force in prestressed concrete box girder bridge using ultrasonic technology" Theses submitted to school of civil engineering and built environment science, faculty of engineering, Queensland University of Technology (2018).

[6] Gandhi, N., Michael, J. E and lee, S. J., " Acoustoelastic Lamb wave propagation in biaxially stressed plates" 2012 Acoustical Society of America 132: 1284. https://doi.org/10.1121/1.4740491.

[7] Qui, L., Yan, X., Lin, X., Yuan, S., "Multiphysics simulation method of lamb wave propagation with piezoelectric transducers under load condition" Chinese Journal of Aeronautics (2019), 32(5): 1071-1086.

[8] Shi, F., Michael, J. E., Lee, S. J., "An ultrasonic guided wave method to estimate applied biaxial loads" AIP Conf. Proc. 1430, 1567-1574(2012); doi:10.1063/1.4716401.

[9] Mohabuth, M., Kotousov, A., Ng, C.T., "Effect of uniaxial stress on the propagation of higherorder lamb wave modes" International journal of Non Linear Mechanics 86 (2016) 104-111.

[10] Lim, H. J., Sohn, H., "Online Stress Monitoring Technique Based on Lamb-wave Measurements and a Convolutional Neural Network Under Static and Dynamic Loadings" Experimental Mechanics (2020) 60:171-179. 